\title{
Coverage Analysis of CR-based Satellite-Terrestrial NOMA Networks with Practical System Impairments
}

\author{
Yerassyl Akhmetkaziyev, Galymzhan Nauryzbayev, ${ }^{\circ}$ Sultangali Arzykulov, ${ }^{\circ}$ Ahmed M. Eltawil, and ${ }^{\ddagger}$ Theodoros A. Tsiftsis \\ School of Engineering and Digital Sciences, Nazarbayev University, Nur-Sultan, Kazakhstan \\ ${ }^{\circ}$ Computer, Electrical, and Mathematical Sciences \& Engineering Division, KAUST, Thuwal, KSA 23955-6900 \\ ¥ The School of Electrical and Information Engineering, and Institute of Physical Internet, Jinan University, China \\ Email: \{yerassyl.akhmetkaziyev, galymzhan.nauryzbayev\}@nu.edu.kz, \\ ○\{sultangali.arzykulov@gmail.com, ahmed.eltawil@kaust.edu.sa\}, ${ }^{\ddagger}$ theo_tsiftsis@jnu.edu.cn
}

\begin{abstract}
In this paper, we investigate a non-orthogonal multiple access (NOMA) assisted cognitive satellite-terrestrial network under practical system conditions, such as transceiver hardware impairments, channel state information mismatch, imperfect successive interference cancellation and interference noises. Generalized coverage probability formulas for NOMA users in both primary and secondary networks are derived considering the impact of interference temperature constraint. Moreover, the numerical results demonstrate superior outperformance compared to the ones obtained for an orthogonal multiple access scheme. Finally, the derived analytical findings are fully supported by thorough Monte Carlo simulations.
\end{abstract}

Index Terms-Cognitive radio (CR), coverage probability, nonorthogonal multiple access (NOMA), satellite-terrestrial networks.

\section{INTRODUCTION}

C OGNITIVE satellite-terrestrial networks (CSTNs) have been gaining much attention in recent years from academia and industry due to their capability of providing a stable data connection with a wide coverage area, including rural and isolated areas, where the allocation of terrestrial infrastructures are profligate or challenging. Moreover, the integration with cognitive radio (CR) technique allows CSTNs to solve critical issues associated with spectral congestion since it enables spectrum sharing between satellite and terrestrial networks [1]. Thus, the CSTNs have been considered as a rising facilitator of future generation wireless networks [2].

On the other hand, due to the constantly expanding number of wireless devices, it is foreseeable that massive connectivity will become a major criteria for CSTNs in order to satisfy the requirements of fifth generation $(5 \mathrm{G})$ and beyond wireless networks. Traditionally, satellite-terrestrial systems mainly adopt orthogonal multiple access (OMA) scheme to provide services. However, it is considered as one of the main bottlenecks since there is a limitation in the number of servable devices due to its orthogonality. As a remedy, non-orthogonal multiple access (NOMA) technique has been recently exploited as a key technology in the future wireless networks because of its ability to serve multiple users on the same time/frequency/code resources while differentiating the users on the base of different power levels [3]. Therefore, a novel NOMA scheme has attracted significant interest from research [4], [5]. For instance, the authors in [4], evaluated the performance of downlink NOMA transmission in millimeter-wave networks in terms of coverage probability $(\mathrm{CP})$. Furthermore, in [5], the authors evaluated the outage performance of CR-NOMA by considering an amplifyand-forward relaying scheme, while a similar system model was examined considering the detect-and-forward scheme [6].

However, until now, very few works have investigated the performance of NOMA in CSTNs [7]-[9]. For instance, the authors in [7] derived the closed-form outage probability (OP) and approximated ergodic capacity expressions for the primary and secondary users for a decode-and-forward relay protocol.

In contrast to [7]-[9], this paper examines the NOMAbased CSTN considering the aggregate transceiver distortions, channel and successive interference cancellation (SIC) imperfections, hardware impairment (HI) as well as interference temperature constraint (ITC). Hence, the key contributions of this paper are as follows. First, closed-form analytical expressions for the CP of the proposed NOMA-assisted CSTN is derived for both primary and secondary networks. Second, the influence of several system parameters on the network performance is investigated using derived analytical results, and, by comparing the NOMA users' CP performance with a benchmark OMA scheme, the advantage of NOMA is verified. Finally, the correctness of derived analytical findings are validated by thorough Monte Carlo simulations.

\section{SySTEM MODEL}

Consider a downlink underlay multi-user CSTN model consisting of primary and secondary networks as illustrated in Fig. 1. The satellite primary network (SPN) comprises the primary satellite transmitter $(T)$ which intents to directly communicate with multiple primary users (PUs), indicated by $R_{n}, n \in\{1,2, \ldots, N\}$. At the same time, the terrestrial secondary network (TSN) consists of a secondary transmitter $(S)$, which can access the licensed band spectrum of PUs and communicate with the secondary users (SUs), denoted by $U_{k}, k \in\{1,2, \ldots, K\}$. In this case, the PUs can suffer from the aggregate interference from $S$ while $T$ interferes the SUs.

\section{A. Channel Models}

We model the communication links using linear minimum mean square error channel estimator as [10],

$$
\chi=\tilde{\chi}+\epsilon,
$$


where $\chi$ denotes the observed mismatched channel, while $\tilde{\chi}$ is real channel estimate and $\epsilon$ indicates its estimation error, with $\mathcal{C N}(0, \lambda)$, where $\lambda$ can be described considering the nominal transmit SNR $\omega$, as $\lambda=\Phi \omega^{-\eta}$. The perfect CSI can be obtained if $\eta \rightarrow \infty$ for $\omega>0$. Moreover, additive white Gaussian noise (AWGN), with mean zero and variance $\sigma^{2}$, exposes all the receiving nodes.

1) Satellite Direct and Interference Channels: The channels corresponding to the satellite links given by $\mathbf{g}_{T U}=$ $\left[g_{T 1}, g_{T 2}, \ldots, g_{T K}\right]$ and $\mathbf{h}_{T R}=\left[h_{T 1}, h_{T 2}, \ldots, h_{T N}\right]$ follow shadowed-Rician fading. Thus, the corresponding probability density function (PDF) is given by [11]

$$
f_{\left|\chi_{n}\right|^{2}}(x)=\Upsilon x^{\ell} e^{-\partial_{n} x}, x \geq 0,
$$

with $\Upsilon=\frac{1}{2 \bar{b}_{n}}\left(\frac{2 \bar{b}_{n} m_{n}}{2 \bar{b}_{n} m_{n}+\Omega_{n}}\right)^{m_{n}} \sum_{\ell=0}^{m_{n}-1} \frac{\left(1-m_{n}\right)_{\ell}\left(-\delta_{n}\right)^{\ell}}{(\ell !)^{2}}$ and $\partial_{n}=\varrho_{n}-\delta_{n}$, where $\delta_{n}=\frac{\Omega_{n}}{2 \bar{b}_{n}\left(2 \bar{b}_{n} m_{n}+\Omega_{n}\right)}, \varrho_{n}=\frac{1}{2 \bar{b}_{n}}$. Here, $2 \bar{b}_{n}$ is the average power of a multi-path component, $\Omega_{n}$ denotes the average power of a line-of-sight (LoS) component, and $m_{n}$ represents the Nakagami parameter. Furthermore, as the Friis' law for free-space propagation states, the pathloss for satellite communication links have linear dependence with logarithmic distance as in [12] and can be expressed by $L(D)=\frac{1}{\mathcal{K}_{\mathcal{B}} \mathcal{T} \mathcal{W}}\left(\frac{c}{4 \pi f_{c} D}\right)^{2}$, where $D$ represents the distance between $T$ and the user of interest ${ }^{1}$, while $c$ and $f_{c}$ denote the light speed and carrier frequency, respectively. $\frac{1}{\mathcal{K}_{\mathcal{B}} \mathcal{T} \mathcal{W}}$ is a scale factor, where $\mathcal{K}_{\mathcal{B}}, \mathcal{T}$ and $\mathcal{W}$ denote the Boltzmann's constant, receiver noise temperature and carrier bandwidth, respectively.

We denote the antenna gain at $T$ as $G_{T}$, with $\varphi$ representing the angle between the $i$-th user's location and the beam center with respect to the satellite. Hence, the beam gain $G_{i}(\varphi)$ is expressed as [14] $G_{i}(\varphi)=G_{i, \max }\left(\frac{J_{1}(u)}{2 u}+36 \frac{J_{3}(u)}{u^{3}}\right)^{2}$, where $u=2.07 \frac{\sin \varphi}{\sin \varphi_{3 \mathrm{~dB}}}, \varphi_{3 \mathrm{~dB}}$ is the constant $3-\mathrm{dB}$ angle for the beam and $J_{l}(\cdot)$ express the first-kind Bessel function of order $l$.

2) Terrestrial Direct and Interference Channels: The terrestrial links given by $\mathbf{g}_{S U}=\left[g_{S 1}, g_{S 2}, \ldots, g_{S K}\right]$ and $\mathbf{h}_{S R}=$ $\left[h_{S 1}, h_{S 2}, \ldots, h_{S N}\right]$ are presumed to abide the Nakagami- $m$ fading. Hence, the channel gains (i.e., squared values) are Gamma random variables (RVs) with the PDF given by

$$
f_{\left|\chi_{k}\right|^{2}}(y)=\frac{y^{m_{k}-1} e^{-\frac{y}{\nu}}}{\Gamma\left(m_{k}\right) \nu^{m_{k}}}
$$

where $m_{k}$ and $\nu$ stand for the shape and scale parameters.

In addition, the sectored antenna pattern has been used to model analog beamforming between communicating nodes as $G(\theta)=G_{m}$, when $\theta \leq \theta_{b}$; otherwise, $G(\theta)=G_{s}$, where $G_{m}$ and $G_{s}$ denote the main and side lobe gains ${ }^{2}$, respectively. $\theta$ is the angle of a boresight direction, while $\theta_{b}$ indicates the antenna beamwidth. For the convenience, we assume that the terrestrial station interferes the PUs with side lobe gain, while

\footnotetext{
${ }^{1}$ Without loss of generality, it is assumed that the distances between the satellite and terrestrial users are equal.

${ }^{2}$ It is assumed that an antenna gain has a constant value in the frame of given main or side lobe sectors.
}

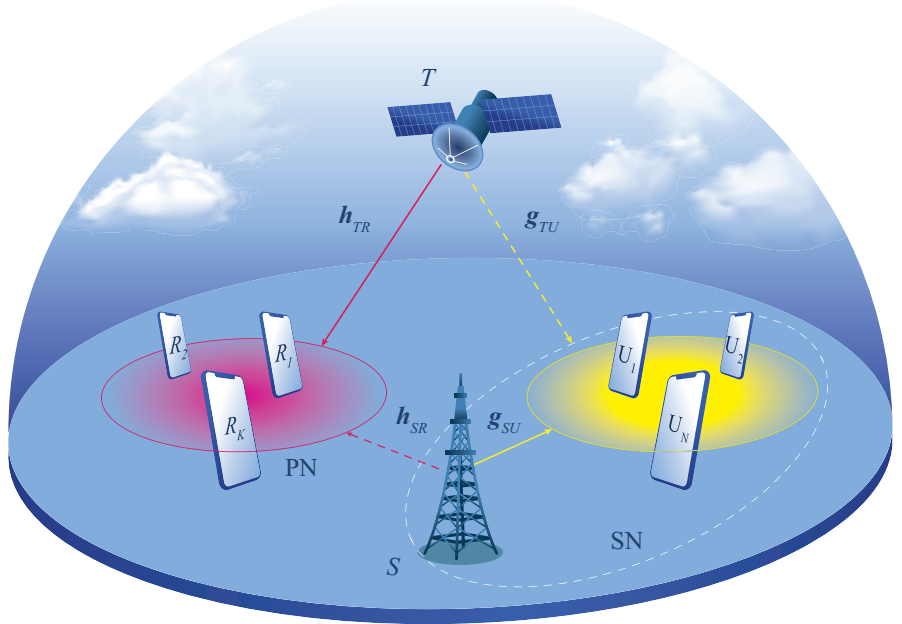

Fig. 1. NOMA-assisted CSTN model.

main lobe gain is exploited in the terrestrial direct links.

\section{B. Signal and SINR Models}

1) Satellite Primary Network: The satellite $T$ and $N$ terrestrial end-users $\left(R_{n, n \in\{1, \ldots, N\}}\right)$ comprise the downlink SPN that employs the NOMA concept by broadcasting a superposed signal $x=\sum_{n=1}^{N} \sqrt{\alpha_{n}} x_{n}$, where $x_{n}$ and $\alpha_{n}$ denote the message devoted for the $n$-th user and the corresponding power allocation (PA) coefficient (with $\alpha_{1}>\alpha_{2}>\ldots>\alpha_{N}$ s.t. $\left.\sum_{n=1}^{N} \alpha_{n}=1\right)$.

Hence, for the sake of brevity and considering non-ideal SIC, the signal-to-interference-noise-distortion ratio (SINDR) at $R_{i}$ to decode message $x_{n}$, for $n \leqslant i$, can be written by arranging $\rho_{i}=P_{T} G_{T} G_{i}(\varphi) L, \bar{\rho}_{i}=P_{S} G_{S} G_{i} d_{S R i}^{-\tau}, X_{i}=\left|\tilde{h}_{T R_{i}}\right|^{2}, Y_{i}=$ $\left|h_{S R_{i}}\right|^{2}, a=\alpha_{n} \rho_{i}$, as follows

$$
\gamma_{i \rightarrow n}=\frac{a X_{i}}{b X_{i}+\Sigma_{i}+c Y_{i}},
$$

where $\Sigma_{i}=\rho_{i}\left(1+\kappa_{i}^{2}\right) \sigma_{\epsilon}^{2}+\sigma_{i}^{2}$ stands for the power levels of channel error (i.e., $\sigma_{\epsilon}^{2}$ ) and AWGN noise (i.e., $\sigma_{i}^{2}$ ), respectively. The interference received from $S$ is given by $c=\left(1+\bar{\kappa}_{S R_{i}}^{2}\right) \bar{\rho}_{i} \cdot b=\rho_{i} \mathcal{A}$ represents the SIC-based interference, with $\mathcal{A}=\left(\Psi_{n}+\tilde{\Psi}_{n}+\kappa_{i}^{2}\right)$, where $\Psi_{n}=\sum_{t=n+1}^{N} \alpha_{t}$, and $\tilde{\Psi}_{n}=\sum_{l=1}^{n-1} \xi_{l} \alpha_{l}$, with $0 \leq \xi \leq 1$ [15]. $\kappa_{i}$ and $\bar{\kappa}_{i}$ denote the compound HI levels observed in the transmission sessions between the respective transceivers. Note that $R_{1}$ decodes its own message by considering messages' of the other users as a noise and defining $\Psi_{1}=\sum_{t=2}^{N} \alpha_{t}$ and $\tilde{\Psi}_{1}=0$. On the other hand, only $R_{N}$ needs to decode message $x_{N}$, subject to $\Psi_{N}=0$ and perfect/imperfect SIC realizations given by $\tilde{\Psi}_{n}=\sum_{l=1}^{n-1} \xi_{l} \alpha_{l}$.

2) Terrestrial Secondary Network: On the other hand, the TSN consists of the secondary transmitter $S$ and $K$ NOMA end-users $\left(U_{k, k \in\{1, \ldots, K\}}\right)$, when the superimposed signal $s=$ $\sum_{k=1}^{K} \sqrt{\beta_{k}} s_{k}$ is sent to all intended SUs, where $s_{k}$ and $\beta_{k}$ indicate the message dedicated for the $k$-th $\mathrm{SU}$ and the corresponding PA coefficient (with $\beta_{1}>\beta_{2}>\ldots>\beta_{K}$ 
s.t. $\left.\sum_{k=1}^{K} \beta_{k}=1\right) . G_{S}$ is the antenna gain at $S$. In the same manner, we can write the SINDR for $U_{j}$, for $j \in\{1, \ldots, K\}$, to detect message $s_{k}$ from $S$ as follows

$$
\psi_{j \rightarrow k}=\frac{W_{1} Z_{j} P_{S}}{P_{S}\left(W_{2} Z_{j}+E\right)+C Q_{j}+\sigma_{j}^{2}},
$$

where $Z_{j}=\left|\tilde{g}_{j}\right|^{2}, Q_{j}=\left|g_{T_{j}}\right|^{2}$, and $W_{1}=\beta_{k} G_{S} G_{D_{j}} d_{j}^{-\tau}$. The power of channel error and AWGN noise is denoted by $E=$ $G_{S} G_{D_{j}} d_{j}^{-\tau}\left(1+{\overline{\kappa_{i}}}^{2}\right) \sigma_{\epsilon}^{2} . W_{2}=G_{S} G_{D_{j}} d_{j}^{-\tau} \overline{\mathcal{A}}$ stands for the SIC-based interference, with $\overline{\mathcal{A}}=\left(\Psi_{j}+\tilde{\Psi}_{j}+\bar{\kappa}_{i}^{2}\right)$, whereas $C=\left(1+\kappa_{T_{j}}^{2}\right) P_{T} G_{T} G_{D}(\varphi) L$ denotes the interference term received from $T$.

\section{Coverage Probability}

\section{A. Satellite Primary Network}

By definition, the coverage is defined as the probability that the SINDR value greater than a predefined signal-to-noise ratio (SNR) associated rate threshold, which can be expressed as $v=2^{\mathcal{R}}-1$, where $\mathcal{R}$ is the data rate threshold [13].

Thus, using (4), the CP of decoding message $x_{n}$ by $R_{i}$ can be determined as

$$
\mathbb{P}_{\text {cov }, i}(v)=\left[\gamma_{i \rightarrow n}>v\right], 0<i \leq n .
$$

Proposition 1: Considering imperfect SIC/CSI and HI, we can express the closed-form expression of the $\mathrm{CP}$ for the primary user of interest as in (7).

Proof: Derivation details are drawn in Appendix A.

\section{B. Terrestrial Secondary Network}

The CP for $U_{K}$ can be expressed using (5) and considering ITC as in (8), where $S_{i}=\frac{I_{\mathrm{ITC}}^{[i]}}{Y_{i}}$ and $P_{S}=\min \left(\bar{P}_{S}, S^{*}\right)$ by assuming $S^{*}=\min \left(S_{1}, S_{2}, \ldots, S_{N}\right)$. Further, we denote $I_{\mathrm{ITC}}^{[i]}=I_{\mathrm{ITC}} d_{S R_{i}}^{\tau}$ and $Y_{i}=\left|h_{S R_{i}}\right|^{2}$.

For the convenience, we simplify the expression by using the following definitions: $T_{1}=\frac{v\left(E P_{s}+\sigma_{j}^{2}\right)}{P_{s}\left(W_{1}-v W_{2}\right)}, T_{2}=$ $\frac{v C}{P_{s}\left(W_{1}-v W_{2}\right)}, J_{1}=\frac{v \sigma_{j}^{2}}{I_{\mathrm{ITC}}^{[i]}\left(W_{1}-v W_{2}\right)}, J_{2}=\frac{v C}{I_{\mathrm{ITC}}^{[i]}\left(W_{1}-v W_{2}\right)}, J_{3}=$ $\frac{v I_{\mathrm{ITC}}^{[i]} E}{I_{\mathrm{IT} T}^{[i]}\left(W_{1}-v W_{2}\right)}, \Lambda=\frac{I_{\mathrm{ITC}}^{[i] \mathrm{C}}}{P_{s}}$.

Proposition 2: The CP in the TSN given by (8) can be expressed in closed form considering ITC as sum of (9) and (10), where, for the sake of brevity, the terms $A$ and $B$ are demonstrated separately.

$$
\begin{aligned}
A=1-\left(\Upsilon \ell ! \partial_{n}^{-\ell}\right. & -1 \\
& \left.\times\left(\frac{T_{2}}{\nu_{0}}+\partial_{n}\right)^{-n-\ell-1}\right) \frac{\gamma\left(m_{k}, \frac{\Lambda}{\nu}\right)}{\Gamma\left(m_{k}\right)} .
\end{aligned}
$$

Proof: A full derivation can be found in Appendix B.

\section{Numerical RESUlts}

This section provides the numerical results on the $\mathrm{CP}$ for the system model under consideration designated by the simulation
Table I. Simulation parameters.

\begin{tabular}{|l|c|}
\hline Parameter & Value \\
\hline \hline Terrestrial channel parameter, $m_{0}$ & 4 \\
\hline Satellite channel parameters, $\left\{m_{i}, b_{i}, \Omega_{i}\right\}$ & $\{5,0.251,0.279\}$ \\
\hline Terrestrial antenna gains, $\left\{G_{m}, G_{s}\right\}$ & $\{12,-1.1092\} \mathrm{dB}$ \\
\hline Satellite antenna gains, $\left\{G_{T}, G_{i, \max }\right\}$ & $\{4.8,54\} \mathrm{dB}$ \\
\hline Threshold, $\left\{\zeta_{N O M A}, \zeta_{O M A}\right\}$ & $\{3,12\} \mathrm{dB}$ \\
\hline PA factors, $\left\{\alpha_{1}, \alpha_{2}\right\}$ & $\{0.7,0.3\}$ \\
\hline Path-loss exponent, $\tau$ & 2 \\
\hline Orbit height, $D$ & $35786 \mathrm{~km}$ \\
\hline ST-to- $R_{N}$ distance, $\left\{d_{R_{1}}, d_{R_{2}}\right\}$ & $\{150,75\} \mathrm{m}$ \\
\hline ST-to- $U_{K}$ distance, $\left\{d_{U_{1}}, d_{U_{2}}\right\}$ & $\{100,50\} \mathrm{m}$ \\
\hline 3dB angle, $\varphi_{3 d B}$ & $0.4^{\circ}$ \\
\hline Carrier frequency, $f_{c}$ & $2 \mathrm{GHz}$ \\
\hline Interference noise power & $10 \mathrm{~dB}$ \\
\hline Temperature, $\mathcal{T}$ & $300 \mathrm{~K}$ \\
\hline Carrier bandwidth, $\mathcal{W}$ & $15 \mathrm{MHz}$ \\
\hline
\end{tabular}

framework drawn in Table I. We also verify our derived analytical expressions through Monte Carlo simulations. For the sake of simplicity, we consider only two PUs and two SUs ${ }^{3}$.

In Fig. 2, the CP comparison of the analytical NOMA network with simulated OMA scheme for both users with two different levels of ITC is demonstrated. It is seen from the figure that a better CP for the NOMA-based networks is achieved at higher value of the ITC. On the other hand, when a smaller ITC is imposed, the CP degrades by showing a saturation at lower SNR values. Moreover, it is seen that the NOMA-based network outperforms the OMA in terms of CP. However, one can observe that for $U_{1}$ the coverage saturation for the NOMAassisted network starts at the lower levels of transmitting SNR in contrast to the SNR rate for the OMA network. It can be explained by the ITC equation, where the transmit power of $0.5 P_{s}$ increases the level of the ITC impact at $S$.

Fig. 3 shows the impact of CSI mismatch for two users in the TSN. Moreover, it considers the SNR-independent/dependent CSI scenarios. When $\eta=0$, meaning that the channel error variance is independent of the transmit SNR, it is observed that $\Phi$ significantly affects the system performance. Noticeably, $\Phi$ does not have any effect on the CP performance at low SNR values. Particularly, it starts influencing $U_{1}$ distinctively at $35 \mathrm{~dB}$ and $U_{2}$ at $25 \mathrm{~dB}$, approximately. It happens due to the fact that when $\Phi$ approaches 0 , the CSI quality tends to become perfect. Therefore, the small mismatch in CSI does not affect the performance at low SNR. Contrarily, when $\eta \neq 0$, CSI becomes SNR-dependent and achieves saturation only at a greater rate of SNR in comparison with the SNR-independent CSI. One can observe that the increase of $\eta$ leads to the improvement of system performance. Similarly, in case of equal $\eta$ values, the growth of $\Phi$ also leads to a slight improvement of $\mathrm{CP}$ performance. However, it is apparent that $\eta$ has much more influence on the performance than $\Phi$. It is worth mentioning that, despite the overall system improvement, all curves level out on the same CP rate at high SNR values.

\footnotetext{
${ }^{3}$ The usage of multiple NOMA nodes can be impractical in the real-time communication due to the fact that, at a receiver side, the processing complexity of the SIC increases non-linearly by raising the number of users [18]. In addition, the issue becomes more crucial when the SIC error exists [15].
} 


$$
\begin{gathered}
\mathbb{P}_{\mathrm{cov}, i}^{\mathrm{SPN}}(v)=1-\frac{\Upsilon \ell !}{\partial_{n}^{\ell+1}}-\sum_{p=0}^{\ell} \frac{\ell !}{p !} \frac{\Upsilon e^{-I(i) \Sigma_{i}\left(\partial_{n}\right)}}{\partial_{n}^{\ell-p+1}} \sum_{t=0}^{p} \frac{(I(i))^{p} p !\left(\Sigma_{i}\right)^{p-t} c^{t}}{t !(p-t) !} \frac{\Gamma\left(t+m_{k}\right)}{\Gamma\left(m_{k}\right) \nu^{m_{k}}}\left(\frac{1}{\nu}+I(i) \partial_{n} c\right)^{-t-m_{k}} \\
\mathbb{P}_{\mathrm{cov}, \mathrm{j}}^{\mathrm{TSN}}(v)=\operatorname{Pr}\left(\frac{W_{1} Z_{j} P_{S}}{P_{S}\left(W_{2} Z_{j}+E\right)+C Q_{j}+\sigma_{j}^{2}}>v, \bar{P}_{s}<S_{i}\right)+\operatorname{Pr}\left(\frac{W_{1} Z_{j} S_{i}}{S_{i}\left(W_{2} Z_{j}+E\right)+C Q_{j}+\sigma_{j}^{2}}>v, \bar{P}_{s}>S_{i}\right) \\
=\underbrace{\operatorname{Pr}\left(Z_{j}>\left(T_{1}+T_{2} Q_{j}\right), Y_{i}<\Lambda\right)}_{A}+\underbrace{\operatorname{Pr}\left(Z_{j}>\left(J_{1} Y_{i}+J_{2} Q_{j} Y_{i}+J_{3}\right), Y_{i}>\Lambda\right)}_{B} .
\end{gathered}
$$

$$
\begin{aligned}
& B=1-\frac{\Gamma\left(m, \frac{\Lambda}{\nu}\right)}{\Gamma(m)} \Upsilon \ell ! \partial_{n}^{-\ell-1}-e^{\frac{-J_{3}}{\nu_{0}}} M_{B} \sum_{i=0}^{t}\left(\begin{array}{l}
t \\
i
\end{array}\right) J_{1}^{t-i} J_{2}^{i} \frac{\Gamma(t+m)}{\Gamma(m) \nu^{m}} e^{-\left(\frac{1}{\nu}+\frac{J_{1}}{\nu_{0}}\right) \Lambda} \sum_{j=0}^{t+m-1} \frac{\Lambda^{j} \Upsilon}{j !} \\
& \times \frac{V_{2} \Omega^{-(i+\ell+1)}}{\Gamma(-j+t+m)} G_{1,2}^{2,1}\left(\frac{\frac{J_{2} \Lambda}{\nu_{0}}+\partial_{n}}{\Omega} \mid \begin{array}{l}
1-(i+\ell+1) \\
0,-(i+\ell+1)+(-j+t+m)
\end{array}\right)
\end{aligned}
$$

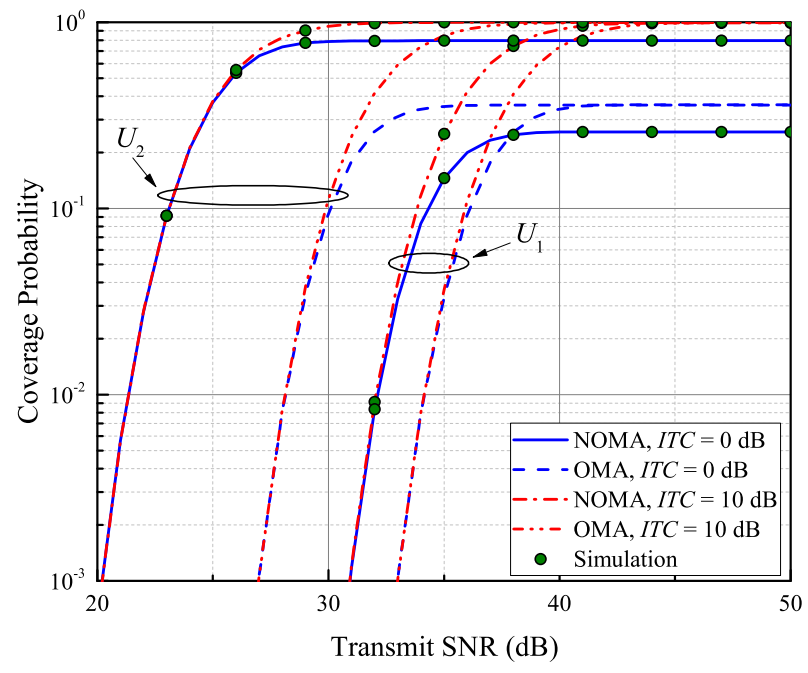

Fig. 2. The CP comparison of the NOMA and OMA schemes for the TSN with different $I T C=\{0,10\} \mathrm{dB}$.

Fig. 4 illustrates the influence of the imperfect CSI parameters on the CP performance for the SPN. Particularly, Fig. 4(a) demonstrates the influence of SNR-independent CSI mismatches by adjusting $\eta=0$. It is clearly noticeable that the $\mathrm{CP}$ performance deteriorates with increasing $\Phi$. it is apparent that imperfect CSI has an enormous effect on system performance. However, this influence of $\Phi$ almost invisible at low SNR levels. In its turn, when $\eta \neq 0$, CSI becomes SNR-dependent. Fig. 4(b) illustrates that the increase of $\eta$ leads to the improvement of system performance. Similarly, in case of equal $\eta$ values, the growth of $\Phi$ also leads to the noticeable improvement of $\mathrm{CP}$ performance. However, it is apparent that $\eta$ has much more effect on the performance than $\Phi$.

In Fig. 5, we examine the influence of the different levels of HIs on the CP performance of the SPN and TSN. Particularly, the HI level was set to three different scenarios with $\kappa=\{0,0.1,0.2\}$. As expected, the $\mathrm{CP}$ of both user deteriorates in the presence of HI. It should be noted that HIs have a low

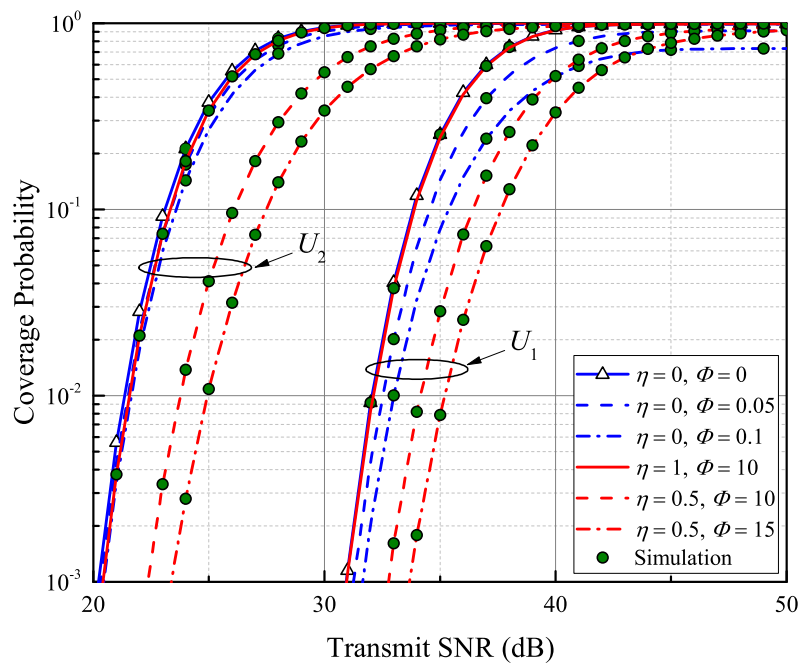

Fig. 3. The CP versus the transmit SNR for the TSN for different CSI scenarios.

impact at low SNRs. Interestingly, a small value of $\mathrm{HI}$ has a marginal impact, especially for user 2 . However, it is still noticeable that a higher rate of HI, i.e., $\kappa=0.2$, can greatly degenerate the overall system performance in terms of the CP.

\section{CONCLUSION}

In this paper, we have evaluated the performance of NOMAassisted CSTNs, wherein the TSN (limited by ITC) co-shares the available spectrum with the SPN. Different from existing works, we have assumed NOMA for both primary and secondary networks and obtained the closed-form CP expressions taking into account the hardware, SIC, and CSI imperfections along with interference noises. Furthermore, a comparison with the OMA network, which is considered as a benchmark scheme, revealed that the proposed NOMA-based CSTN demonstrates outstanding performance advancement while utilizing the spectrum resource in an efficient manner. Finally, Monte Carlo 


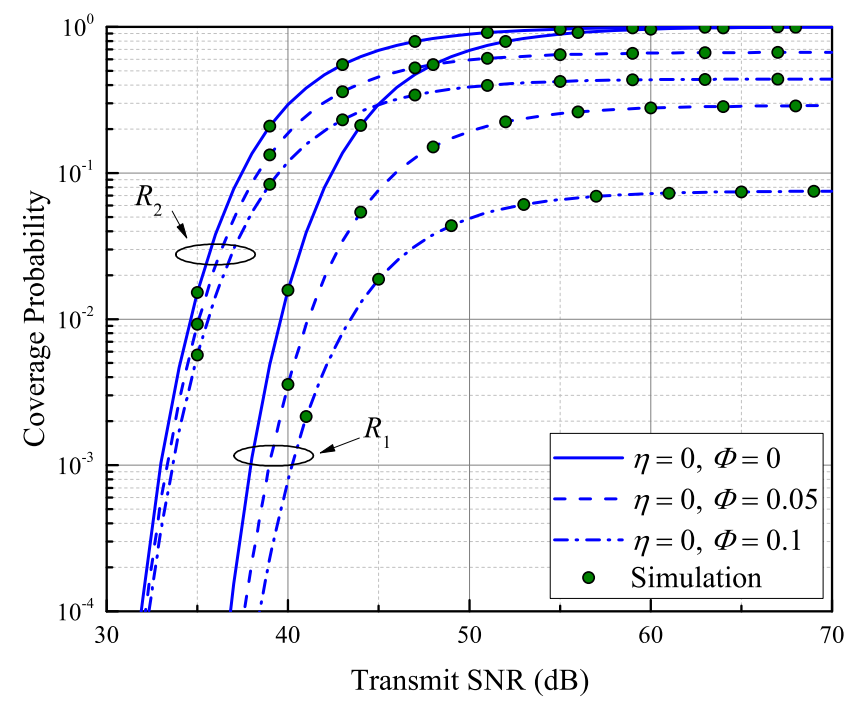

(a) The CP performance for the SPN with perfect CSI and SNR-independent (b) The CP performance for the SPN with SNR-dependent CSI mismatches CSI mismatches $\{0,0.05\}$ and $\{0,0.1\}$.

Fig. 4. The CP versus the transmit SNR for the SPN for different CSI scenarios.

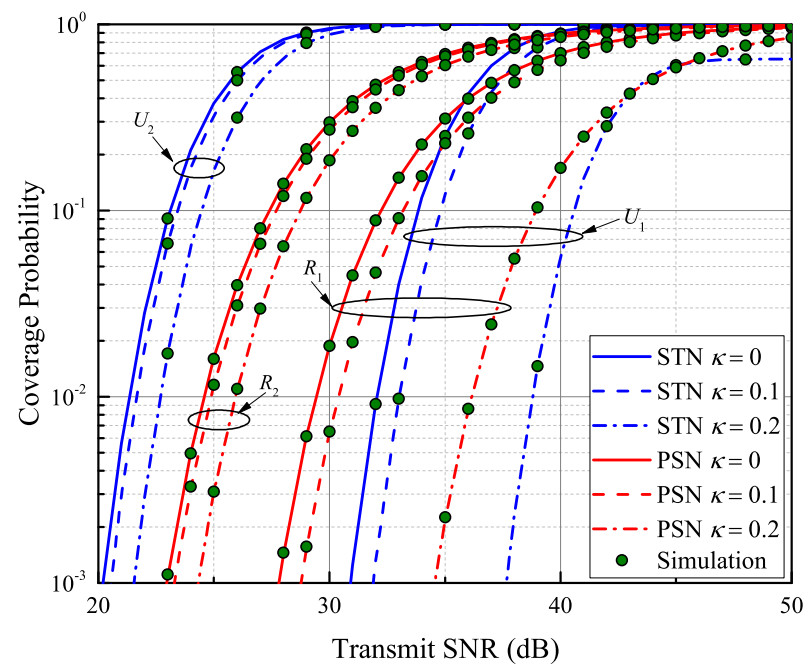

Fig. 5. The CP versus the transmit SNR for the satellite and terrestrial networks with different HI levels.

simulations validated the correctness of the derived analytical findings.

\section{APPENDIX A \\ SATELLITE PRIMARY NETWORK}

In this section, we present the derivation steps for appraising the coverage performance of the NOMA end-user in the SPN.

Hence, the CP can be evaluated using (4) as

$$
\begin{aligned}
& \mathbb{P}_{\mathrm{cov}, i}(v)=\operatorname{Pr}\left[X_{i}>I(i)\left(\Sigma_{i}+c Y_{i}\right)\right] \\
& =1-\int_{0}^{\infty} \underbrace{\left(\int_{0}^{I(i)\left(\Sigma_{i}+c Y_{i}\right)} f_{X_{i}}(x) \mathrm{d} x\right)}_{A_{1}} f_{Y}(y) \mathrm{d} y .
\end{aligned}
$$

where $I(i)=\frac{v}{a_{i}-b_{i} v}$. Here $A_{1}$ can be obtained by integrating

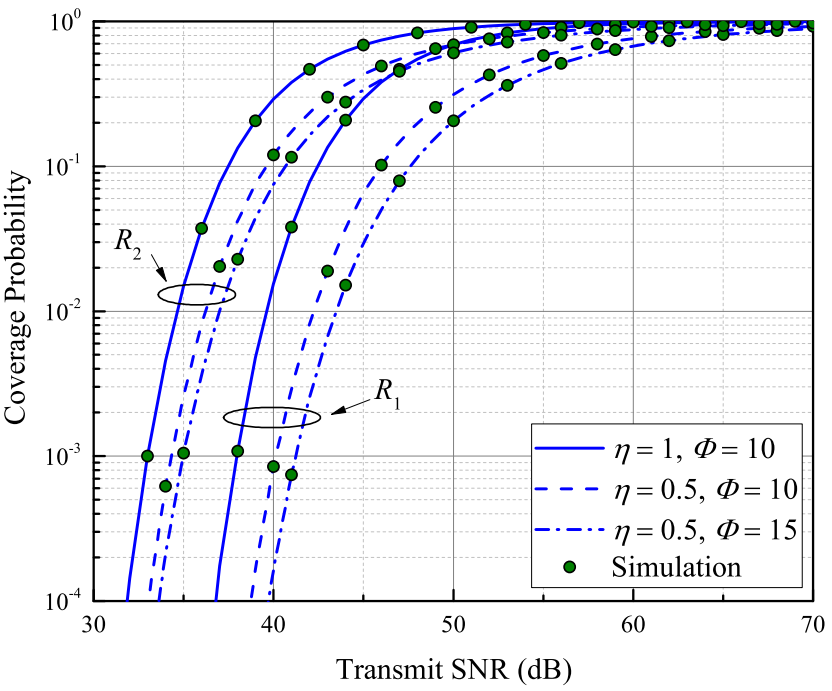

$\{1,10\},\{0.5,10\}$ and $\{0.5,15\}$.

(2) with the aid of [16, Eq. (3.351.1)] as

$$
\begin{aligned}
A_{1}= & \int_{0}^{I(i)\left(\Sigma_{i}+c Y_{i}\right)} \Upsilon x^{\ell} e^{-\partial_{n} x} \mathrm{~d} x=\Upsilon \frac{\ell !}{\partial_{n}^{\ell+1}} \\
& -\Upsilon e^{-I(i)\left(\Sigma_{i}+c Y_{i}\right) \partial_{n}} \sum_{p=0}^{\ell} \frac{\ell !}{p !} \frac{\left(I(i)\left(\Sigma_{i}+c Y_{i}\right)\right)^{p}}{\partial_{n}^{\ell-p+1}} .
\end{aligned}
$$

Further, let us denote $\Delta=I(i)\left(\Sigma_{i}+c Y_{i}\right)$, then $\Delta^{p}$ can be expanded using a binomial theorem as $\Delta^{p}=$ $(I(i))^{p} \sum_{t=0}^{p}\left(\begin{array}{l}p \\ t\end{array}\right)\left(\Sigma_{i}\right)^{p-t}\left(c Y_{i}\right)^{t}$. Now, for the terrestrial interference link, the i.n.i.d. Gamma RVs with shape $m_{k}$ and scale $\nu$ parameters are generated using the PDF in (3). Substituting (12) into (11) and by using $\Delta^{p}$, the CP can be rewritten as

$$
\begin{aligned}
& \mathbb{P}_{\mathrm{cov}, i}(v)=1-\Upsilon \frac{\ell !}{\partial_{n}^{\ell+1}} \int_{0}^{\infty} \frac{y^{m_{k}-1} e^{-\frac{y}{\nu}}}{\Gamma\left(m_{k}\right) \nu^{m_{k}}} \mathrm{~d} y-\sum_{p=0}^{\ell} \frac{\ell !}{p !} \frac{\Upsilon e^{-I(i) \Sigma_{i} \partial_{n}}}{\partial_{n}^{\ell-p+1}} \\
& \times(I(i))^{p} \sum_{t=0}^{p}\left(\begin{array}{l}
p \\
t
\end{array}\right) \frac{\left(\Sigma_{i}\right)^{p-t} c^{t}}{\Gamma\left(m_{k}\right) \nu^{m_{k}}} \int_{0}^{\infty}\left(y_{i}\right)^{t+m_{k}-1} e^{-y\left(\frac{1}{\nu}+I(i) \partial_{n} c\right)} \mathrm{d} y .
\end{aligned}
$$

Finally, we can express the exact CP with the aid of [16, Eq. (8.310.1)] in its closed form as (7).

\section{APPENDIX B}

\section{TERRESTRIAL SECONDARY NETWORK}

The term $A$ in (8) can be rewritten as

$$
A=1-\underbrace{\int_{q=0}^{\infty} f_{Q}(q) \underbrace{\int_{z=0}^{T_{1}+T_{2} Q_{j}} f_{Z}(z) \mathrm{d} z}_{A_{1}}}_{A_{2}} \mathrm{~d} q \underbrace{\int_{y=0}^{\Lambda} f_{Y}(y) \mathrm{d} y}_{A_{3}} .
$$

The PDFs of $Z$ and $Y$ abide by Nakagami- $m$ statistical model, and $Q$ term follows Shadowed-Rician dis- 
tribution. Therefore, by using the PDF in (3), we obtain $A_{1}=1-e^{\frac{-T_{1}-T_{2} Q_{j}}{\nu_{0}}} M_{A}\left(T_{2} Q_{j}\right)^{n}$, where $M_{A}=$ $\sum_{p=0}^{m_{0}-1} \frac{1}{\nu_{0}^{p} \Gamma(p+1)} \sum_{n=0}^{p}\left(\begin{array}{l}p \\ n\end{array}\right) T_{1}^{p-n}$. Now, knowing the results of $A_{1}$ and using the PDF in (2), we can calculate $A_{2}$ with the aid of [16, Eq. (3.351.3)] as

$$
\begin{aligned}
& A_{2}=\left(1-e^{\frac{-T_{1}-T_{2} Q_{j}}{\nu_{0}}} M_{A}\left(T_{2} Q_{j}\right)^{n}\right) \Upsilon \int_{0}^{\infty} q^{\ell} e^{-\partial_{n} q} \mathrm{~d} q \\
& =\Upsilon \ell ! \partial_{n}^{-\ell-1}-e^{\frac{-T_{1}}{\nu_{0}}} M_{A} T_{2}^{n} \Upsilon(n+\ell) !\left(\frac{T_{2}}{\nu_{0}}+\partial_{n}\right)^{-n-\ell-1},
\end{aligned}
$$

and the CDF of $A_{3}$ can be obtained independently as $\frac{\gamma\left(m_{k}, \frac{\Lambda}{\nu}\right)}{\Gamma\left(m_{k}\right)}$. Finally, by inserting $A_{2}$ and $A_{3}$ into (A.1) we can express the term $A$ in a closed-form as (9).

The term $B$ in (8) can be determined as

$$
B=1-\int_{q=0}^{\infty} f_{Q}(q) \underbrace{\int_{y=\Lambda}^{\infty} f_{Y}(y) \int_{0}^{J_{1} Y+J_{2} Y Q_{j}+J_{3}} f_{Z}(z) \mathrm{d} z \mathrm{~d} y}_{B_{1}} \mathrm{~d} q .
$$

Here, using (3) and the CDF of $Z, B_{1}$ can be calculated as

$$
\begin{aligned}
& B_{1}=\left(1-e^{\frac{-J_{1} Y-J_{2} Y Q_{j}-J_{3}}{\nu_{0}}} \sum_{p=0}^{m_{0}-1} \frac{\left(J_{3}+Y\left(J_{1}+J_{2} Q_{j}\right)\right)^{p}}{\nu_{0}^{p} \Gamma(p+1)}\right) \\
& \times \int_{\Lambda}^{\infty} \frac{y^{m-1} e^{-\frac{y}{\nu}}}{\Gamma(m) \nu^{m}} \mathrm{~d} y=\frac{\Gamma\left(m, \frac{\Lambda}{\nu}\right)}{\Gamma(m)}-e^{\frac{-J_{3}}{\nu_{0}}} M_{B}\left(J_{1}+J_{2} Q_{j}\right)^{t} \\
& \times \int_{\Lambda}^{\infty} \frac{y^{t} y^{m-1} e^{-y\left(\frac{1}{\nu}+V_{1} Q_{j}\right)}}{\Gamma(m) \nu^{m}} \mathrm{~d} y=\frac{\Gamma\left(m, \frac{\Lambda}{\nu}\right)}{\Gamma(m)}-e^{\frac{-J_{3}}{\nu_{0}}} M_{B} \\
& \times \underbrace{\left(J_{1}+J_{2} Q_{j}\right)^{t} \frac{\Gamma\left(t+m,\left(\frac{1+\nu V_{1} Q_{j}}{\nu}\right) \Lambda\right)}{\Gamma(m) \nu^{m}}\left(\frac{1+\nu V_{1} Q_{j}}{\nu}\right)^{-t-m}}_{B_{2}} .
\end{aligned}
$$

where $M_{B}=\sum_{p=0}^{m_{0}-1} \frac{1}{\nu_{0}^{p} \Gamma(p+1)} \sum_{t=0}^{p}\left(\begin{array}{l}p \\ t\end{array}\right)\left(J_{3}\right)^{p-t}, V_{1}=\frac{J_{1}+J_{2}}{\nu_{0}}$. Now, we need to apply the series representation of the upper incomplete Gamma function given by [16] as $\Gamma(b, c)=$ $\Gamma(b) e^{-c} \sum_{i=0}^{b-1} \frac{c^{i}}{i !}$ and binomial theorem in order to expand $B_{2}$ in (B.2). Then, by using the aforementioned methods and after some algebraic manipulations, we rewrite $B_{2}$ as

$$
\begin{aligned}
& B_{2}=\sum_{i=0}^{t}\left(\begin{array}{c}
t \\
i
\end{array}\right) J_{1}^{t-i}\left(J_{2} Q_{j}\right)^{i} \sum_{j=0}^{t+m-1} \frac{\Gamma(t+m) \Lambda^{j} V_{2}}{\Gamma(m) \nu^{m} j !} \\
& \times e^{-\left(\frac{1}{\nu}+\frac{J_{1}+J_{2} Q_{j}}{\nu_{0}}\right) \Lambda}\left(1+\Omega Q_{j}\right)^{j-t-m},
\end{aligned}
$$

where $V_{2}=\left(\frac{\nu_{0}+\nu J_{1}}{\nu_{0} \nu}\right)^{j-t-m}, \Omega=\frac{\nu J_{2}}{\nu_{0}+\nu J_{1}}$. Thus, the term $B$ can be rewritten as

$$
\begin{aligned}
& B=1-\frac{\Gamma\left(m, \frac{\Lambda}{\nu}\right)}{\Gamma(m)} \Upsilon \int_{0}^{\infty} q^{\ell} e^{-\partial_{n} q} \mathrm{~d} q-e^{\frac{-J_{3}}{\nu_{0}}} M_{B} \\
& \times \sum_{i=0}^{t}\left(\begin{array}{l}
t \\
i
\end{array}\right) J_{1}^{t-i} J_{2}^{i} \frac{\Gamma(t+m)}{\Gamma(m) \nu^{m}} e^{-\left(\frac{1}{\nu}+\frac{J_{1}}{\nu_{0}}\right) \Lambda} \sum_{j=0}^{t+m-1} \frac{\Lambda^{j}}{j !} V_{2} \Upsilon
\end{aligned}
$$

$$
\times \int_{0}^{\infty}\left(1+\Omega Q_{j}\right)^{-(t+m-j)} q^{i+\ell+1-1} e^{-\left(\frac{J_{2} \Lambda}{\nu_{0}}+\partial_{n}\right) q} \mathrm{~d} q .
$$

Finally, by using the Meijer-G function represented with the aid of [17, Eqs. (7.34.3.46.1) and (7.34.3.271.1)], where $e^{-b \gamma}=$ $G_{0,1}^{1,0}\left(\left.b \gamma\right|_{0} ^{-}\right)$and $(1+c \gamma)^{-d}=\frac{1}{\Gamma(d)} G_{1,1}^{1,1}\left(\left.c \gamma\right|_{0} ^{1-d}\right)$, and after some mathematical manipulations with the additional aid of [16, Eq. (3.351.3)], we obtain the closed-form expression of term $B$ as in (10).

\section{REFERENCES}

[1] Y. Ruan et al., "Spectral-Energy Efficiency Tradeoff in Cognitive Satellite-Vehicular Networks Towards Beyond 5G," 2019 IEEE Wireless Communications and Networking Conference (WCNC), Marrakesh, Morocco, pp. 1-6, 2019.

[2] G. Giambene, S. Kota, and P. Pillai, "Satellite-5G Integration: A Network Perspective," IEEE Network, vol. 32, no. 5, pp. 25-31, Oct. 2018.

[3] M. Liu, T. Song, and G. Gui, "Deep Cognitive Perspective: Resource Allocation for NOMA-Based Heterogeneous IoT With Imperfect SIC," IEEE Internet of Things Journal, vol. 6, no. 2, pp. 2885-2894, Apr. 2019.

[4] Y. Zhou, V. W. S. Wong, and R. Schober, "Coverage and Rate Analysis of Millimeter Wave NOMA Networks With Beam Misalignment," IEEE Transactions on Wireless Communications, vol. 17, no. 12, pp. 8211-8227, Dec. 2018.

[5] S. Arzykulov et al., "Performance Analysis of Underlay Cognitive Radio Nonorthogonal Multiple Access Networks," IEEE Transactions on Vehicular Technology, vol. 68, no. 9, pp. 9318-9322, Sep. 2019.

[6] S. Arzykulov et al., "Underlay Spectrum Sharing for NOMA Relaying Networks: Outage Analysis," 2020 International Conference on Computing, Networking and Communications (ICNC), Big Island, HI, USA, pp. 897-901, 2020.

[7] X. Zhang et al., "Performance Analysis of NOMA-Based Cooperative Spectrum Sharing in Hybrid Satellite-Terrestrial Networks," IEEE Access, vol. 7, pp. 172321-172329, 2019.

[8] V. Singh, P. K. Upadhyay, and M. Lin, "On the Performance of NOMAAssisted Overlay Multiuser Cognitive Satellite-Terrestrial Networks," IEEE Wireless Communications Letters, vol. 9, no. 5, pp. 638-642, May 2020.

[9] X. Yan et al., "On the ergodic capacity of NOMA-based cognitive hybrid satellite terrestrial networks," 2017 IEEE/CIC International Conference on Communications in China (ICCC), Qingdao, 2017, pp. 1-5.

[10] Y. Gao et al., "Analysis of the Dynamic Ordered Decoding for Uplink NOMA Systems With Imperfect CSI," IEEE Transactions on Vehicular Technology, vol. 67, no. 7, pp. 6647-6651, Jul. 2018.

[11] A. Abdi et al., "A new simple model for land mobile satellite channels: first- and second-order statistics," IEEE Transactions on Wireless Communications, vol. 2, no. 3, pp. 519-528, May 2003.

[12] K. Guo et al., "Outage analysis of cognitive hybrid satellite-terrestrial networks with hardware impairments and multi-primary users," IEEE Wireless Communication Letters, vol. 7, no. 5, pp. 816-819, Oct. 2018.

[13] G. Nauryzbayev, M. Abdallah, and K. M. Rabie, "Outage Probability of the EH-Based Full-Duplex AF and DF Relaying Systems in $\alpha-\mu$ Environment," IEEE 88th Vehicular Technology Conference (VTC-Fall), Chicago, IL, USA, 2018, pp. 1-6.

[14] P. K. Sharma, D. Deepthi, and D. I. Kim, "Outage Probability of 3-D Mobile UAV Relaying for Hybrid Satellite-Terrestrial Networks," IEEE Communications Letters, vol. 24, no. 2, pp. 418-422, Feb. 2020.

[15] S. Arzykulov et al., "Hardware- and Interference-Limited Cognitive IoT Relaying NOMA Networks With Imperfect SIC Over Generalized NonHomogeneous Fading Channels," IEEE Access, vol. 8, pp. 72942-72956, 2020.

[16] I. S. Gradshteyn and I. M. Ryzhik, Table of integrals, series, and products. Elsevier/Academic Press, Amsterdam, 7th ed., 2007.

[17] Wolfram, “The wolfram functions site." Last visited on 03/10/2020.

[18] Z. Ding, M. Peng, and H. V. Poor, "Cooperative non-orthogonal multiple access in 5G systems," IEEE Communications Letters, vol. 19, pp. 14621465, Aug. 2015. 